\title{
Cervical Tumor in Patient on Chronic Hemodialysis about a Case \\ Parodis López $\mathrm{Y}^{1, *}$, Monzon $\mathrm{T}^{1}$, Alonso $\mathrm{F}^{1}$, Quintana Viñau $\mathrm{B}^{2}$, Gonzalez $\mathrm{M}^{3}$, Valga $\mathrm{F}^{4}$, Hillebrand $\mathrm{S}^{1}$, Betancor $\mathrm{J}^{1}$, Henríquez $\mathrm{F}^{1}$, and Antón $\mathbf{G}^{1}$
}

${ }^{1}$ Unidad de Hemodiálisis, Centro de Diálisis Avericum, Las Palmas, Spain

${ }^{2}$ Department of Radiology, University Hospital of Gran Canaria Dr Negrin, Las Palmas, Spain

${ }^{3}$ Department of Pathological Anatomy, University Hospital of Gran Canaria Dr Negrin, Las Palmas, Spain

${ }^{4}$ Department of Nephrology, University Hospital of Gran Canaria Dr Negrin, Las Palmas, Spain

*Corresponding author: Parodis López Y, Unidad de Hemodiálisis, Centro de Diálisis Avericum, Las Palmas, Gran Canaria, Spain, E-mail: yanet. parodis@avericum.com

Received: 09 Jun, 2021 | Accepted: 19 Jul, 2021 | Published: 24 Jul, 2021

Citation: López YP, Monzon T, Alonso F, Viñau BQ, Gonzalez M, et al. (2021) Cervical Tumor in Patient on Chronic Hemodialysis about a Case. Int J Nephrol Kidney Fail 7(3): dx.doi.org/10.16966/2380-5498.216

Copyright: (C) 2021 López YP, et al. This is an open-access article distributed under the terms of the Creative Commons Attribution License, which permits unrestricted use, distribution, and reproduction in any medium, provided the original author and source are credited.

\begin{abstract}
Patients with Chronic Kidney Disease on Dialysis (CDRD) have a major risk of developing cancer when compared with the general population [1], thyroid carcinoma being the most common in the first group of patients [2]. The definitive treatment is thyroidectomy, but in patients at risk of recurrence ablative therapy with radioactive iodine I-131 is necessary [2]. We present the clinical case of a 61-year-old patient on hemodialysis with a slow growing anterior cervical tumor studied for more than two years until the diagnosis of papillary thyroid carcinoma. Initially the patient received surgical treatment and is currently awaiting treatment with radioactive iodine I-131.
\end{abstract}

Keywords: Cervical tumor; Chronic renal disease in dialysis; Thyroid carcinoma

\section{Introduction}

We present the clinical case of a 62-year-old male who attended the hospital emergency department in September 2008 because of hypertensive crisis, found to have advanced chronic kidney disease and started hemodiaysis via Central Venous Catheter (CVC).

He was born in Mauritania and had a language barrier. From the start of hemodiaysis, he was diagnosed with secondary hyperparathyroidism, as well as multiple vascular access problems involving the placement of multiple central venous catheters. He had several episodes of bacteraemia with bacterial endocarditis on the tricuspid valve and thrombosis of the superior and inferior vena cava, requiring angioplasty and stenting at both levels. He was assessed by the haematology department, which ruled out autoimmune or associated coagulation disorders, except for a weakly positive lupus anticoagulant.

In September 2017, on physical examination, a cervical tumour of firm consistency was detected in the left inferolateral and anterior region of the neck, associated with signs of venous hypertension in the left upper extremity (LUE) (Figure 1) where he had an immature humerus-cephalic Arterio-Venous Fistula (AVF) and a CVC in the left internal jugular vein.

\section{Diagnostic Procedures}

A space-occupying lesion of undetermined nature is found by cervical ultrasound, which could be of vascular origin. ACT scan with contrast was performed, highlighting a laterocervical mass of $6.6 \times$ $5 \mathrm{~cm}$ in diameter in axial plane and $5 \mathrm{~cm}$ in craniocaudal diameter of polycystic morphology with thick walls and hyper capillary septa. In addition, a $1.8 \times 1.5 \mathrm{~cm}$ hyper dense cranially located solid pole with dystrophic calcifications is described, which displaces the airway and larynx and causes compression and thrombosis of the left jugular vein, and a cytological study is requested (Figure 2).

We decided to change the CVC to the right internal jugular vein and immediately carried out cytological and microbiological studies by means of an FNA-echo of several of the lesions, all of which were negative for malignancy and without microbial growth.

Clinically, the patient had remained stable up to this point without odynophagia, dysphonia or dyspnoea. Analytically, he presented tumour markers and blood proteinogram without significant alterations except for B2 microglobulin: $39.1 \mu \mathrm{g} / \mathrm{ml}$ and an elevation in the heterogeneous gamma globulin fraction (polyclonal). In terms of thyroid function, TSH always remained within the normal range and only months after diagnosis a slightly decreased free T4 hormone $(0.66 \mathrm{ng} / \mathrm{dl})$ was found.

Given the absence of diagnosis and the progressive growth of the cervical tumour, interconsultations were requested with the specialities of Haematology, Otorhinolaryngology (ORL) and the infectious diseases service, ruling out haematological neoplasms, hydatidosis, mycobacterial infections, mycoplasma, rickettsia, Q fever and CMV, among others. 
A new CT scan with contrast and PET-CT scan were also requested, which continued to show the same multiloculated mass with cystic content, but with an increase in size with respect to the previous study of $7.6 \mathrm{~cm} \times 5.8 \mathrm{~cm}$ respectively; no other neoplastic lesions or metastases at another level were found. Despite the diagnostic presumption of a colloid cyst, no connection with the thyroid gland could be seen (Figure 2). Again, a second cervical FNA-CT scan revealed negative cytological and microbiological studies.

Surgical treatment of the lesion was considered, but the patient was a social case, non-compliant with treatment, without family support and with repeated absences from consultations and diagnostic studies, which delayed the procedure. Added to this was the absence of an aetiological diagnosis, with the possibility of a vascular tumour with a vital risk of bleeding given its location.

\section{Evolution}

The patient began with clinical symptoms of dysphonia and dysphagia coinciding with an even greater increase in the size of the tumour (Figure 1), finally attending the emergency department for cervical bleeding in the context of tumour fistulisation to the skin.

On this occasion a new cervical CT scan was performed which showed a marked radiological worsening of the tumour with diameters of approximately $7 \times 13 \times 11 \mathrm{~cm}$ in its PA, T and CC axes respectively (previously $5 \times 5.8 \times 7.6 \mathrm{~cm}$ ) as well as a significant expansion effect, displacing the airway laterally to a greater extent and partially collapsing its lumen at supraglottic level (Figure 2).
For the third time, a FNA-echo was performed on several of the lesions and this time it was positive for malignancy and suggestive of a papillary thyroid carcinoma [Category VI (Bethesda)].

\section{Treatment}

The patient undergoes surgery and a total thyroidectomy is performed.

The pathology report describes the existence of a classic variant papillary thyroid carcinoma, present in both the left hemithyroid dependent tumour mass without lymphovascular or perineural invasion. No evidence of malignancy was found in the right isthmus and thyroid lobe (Figure 3).

Evolutionarily, the patient required surgical reintervention for a left supraclavicular collection with good subsequent evolution, with the only initial sequelae being mild dysphonia.

After months of apparent good evolution, the patient was found to have a recurrence of a tumour in situ associated with cervical and systemic lymph node metastases. In view of these findings, it was decided to reoperate with excision of the recurrent mass and left lymph node emptying without complications.

With more than 3 years of evolution, the patient remains alive, with an acceptable quality of life for the time being and is being monitored by the ORL, endocrinology and nuclear medicine services pending treatment with radioactive iodine I- 131 .

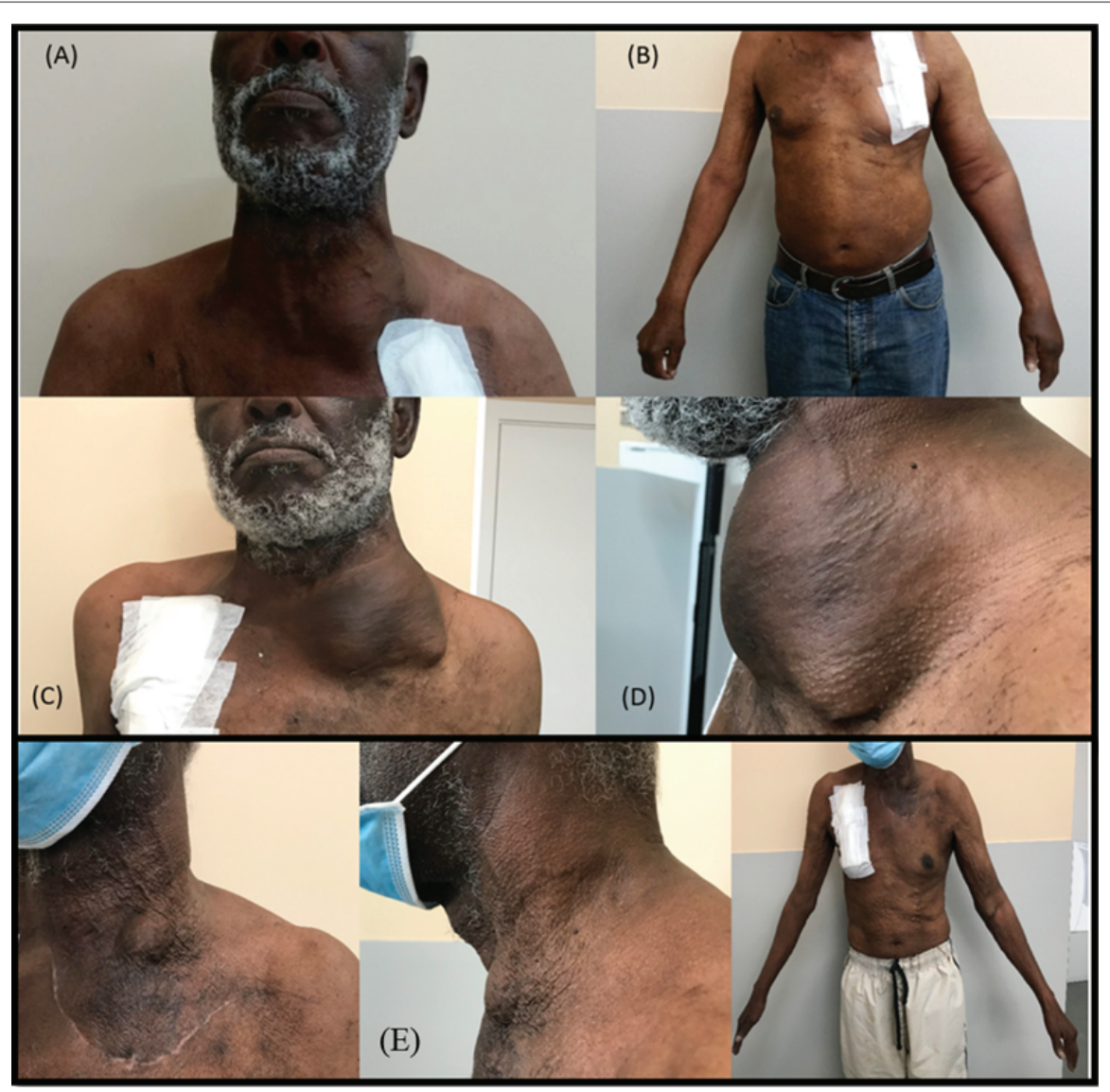

Figure 1: $(A$ and $B)$ : Initial tumor conditioning venous hypertension MSI, (C and D): Cervical tumor 2 years after and before surgery, (E): After excéresis of the tumor that included total thyroidectomy. 


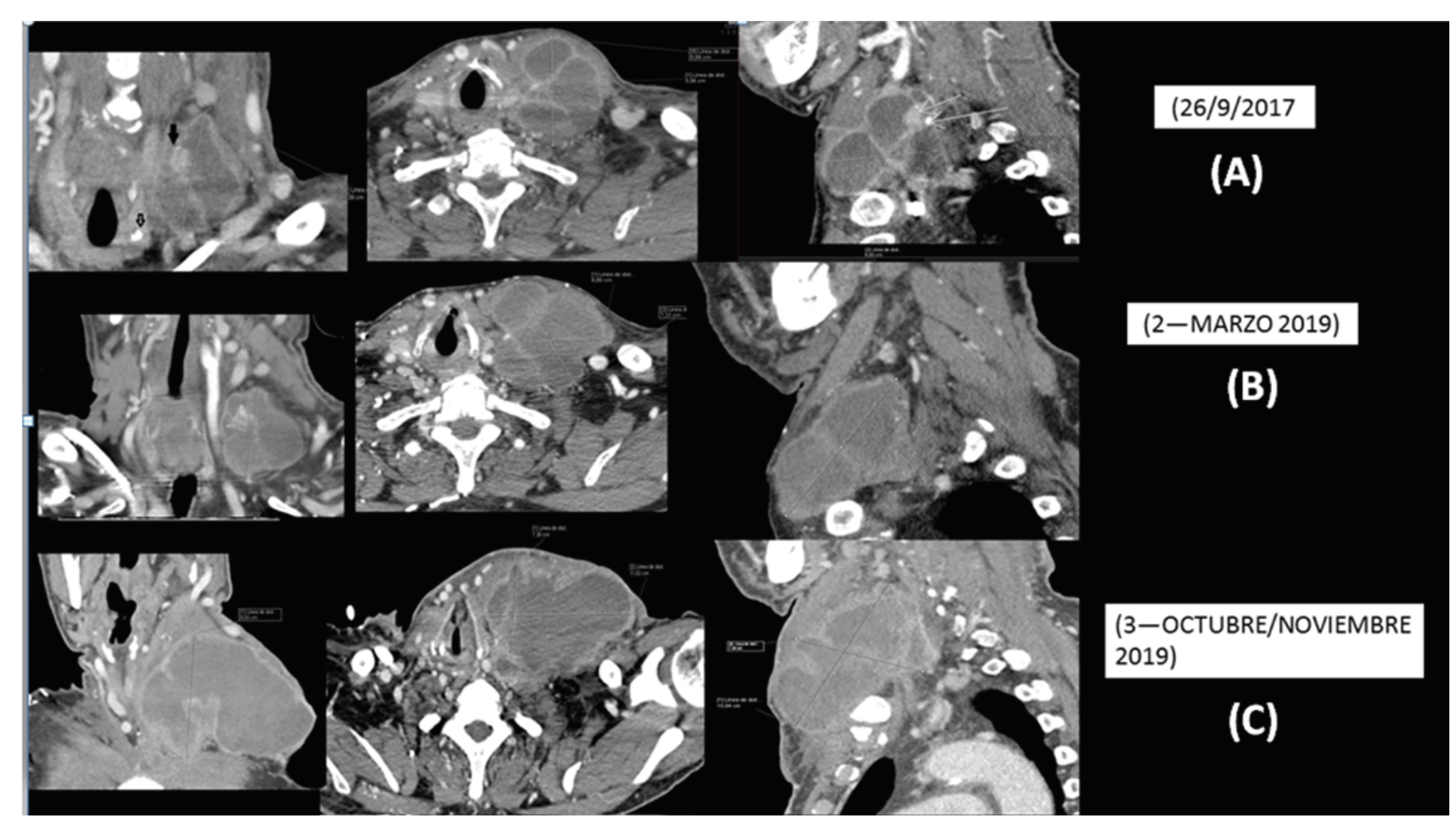

Figure 2: (A): Laterocervical mass measuring $6.6 \times 5 \mathrm{~cm}$ in diameter in axial plane and $5 \mathrm{~cm}$ in craniocaudal diameter with polycystic morphology, ( $B$ and $C$ ): progressive increase in tumour size with displacement of the airway and larynx as well as compression and thrombosis of the left jugular vein.

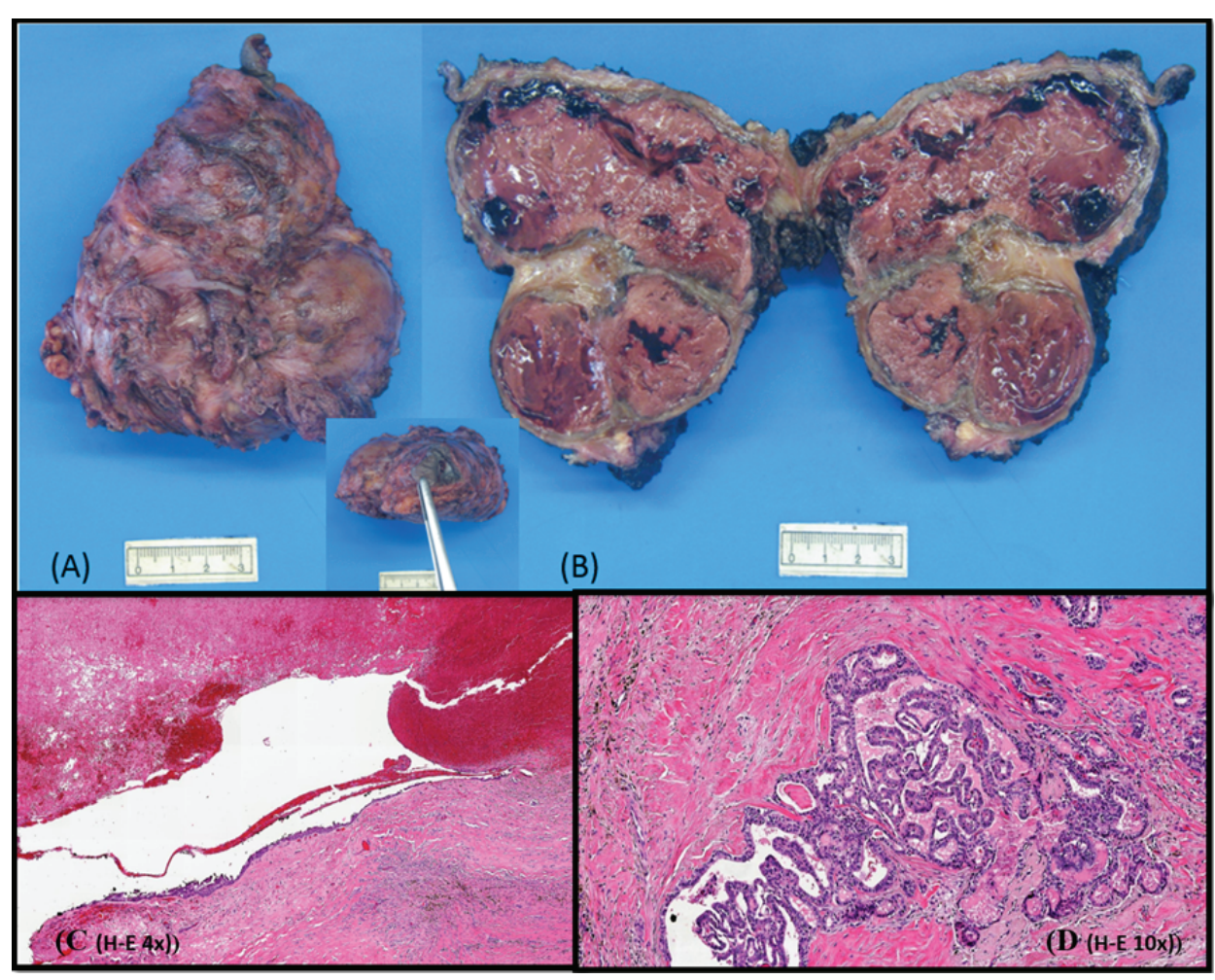

Figure 3: $(A): 11 \times 10 \mathrm{~cm}$ left hemithyroid-dependent tumour mass with central fistulous tract in cutaneous fragment (B): In section, tumour mass with solid whitish septa delimiting lobules of reddish-brown necrotic substance. ( $C$ and D): cystified area of fibrous wall lined with neoplastic cells and papillary carcinoma tumour area in fibrous stroma. 


\section{Discussion and Conclusions}

Patients with chronic kidney disease on dialysis (CKD) have a higher risk of cancer when compared to the general population [1] and in the case of thyroid carcinoma its frequency is higher in the former group of patients [2].

The diagnosis of thyroid cancer in dialysis patients is usually rapid and is often found as an accidental finding in the context of the study of secondary hyperparathyroidism or during the study prior to renal transplantation [2].

In our case, the difficulty of the aetiological diagnosis is striking despite the large size of the tumour, the prolonged time of evolution, as well as the histological studies, imaging tests and multidisciplinary consultations performed.

Papillary thyroid cancer is most frequent between the fourth and fifth decades of life with a higher incidence in women, the ratio being approximately 2.5:1.

A recent study in a haemodialysis population found that the mean time between initiation of haemodialysis and diagnosis of cancer was $64.40 \pm 41.81$ months and the risk factors significantly related to its appearance were: advanced age (especially in patients aged $\geq 70$ years), male sex and liver disease [3].

These data contrast with our male patient who has been on haemodialysis for 12 years, with no history of liver disease, and who was 61 years old at the time of diagnosis.

Cancers derived from the follicular thyroid epithelium are divided into three categories: papillary (85\%), follicular (12\%) and anaplastic (undifferentiated) cancers $(<3 \%)[4]$.

Papillary and follicular cancers are considered differentiated cancers and surgery is the treatment of choice in these patients. Other thyroid malignancies include medullary thyroid cancer (isolated or familial, whether or not part of multiple endocrine neoplasia type 2 [MEN2] syndromes) and primary thyroid lymphoma.

Metastases of other cancers can also be found in the thyroid, the most frequent being breast, colon, renal cancer and melanoma [4].

The histology of our clinical case was compatible with a classic variant of papillary thyroid carcinoma, and given the size and complications, surgical treatment with total thyroidectomy was decided upon due to the doubt of tumour extension to the right hemi thyroid.

Most thyroid cancers have an excellent prognosis and most patients with papillary thyroid cancer do not die from the disease [4].

In a series of patients with a median follow-up of 16 years, the mortality related to this type of cancer in patients without metastases at diagnosis was 6\% [5].

According to the ATA (American Thyroid Association) criteria, our patient initially met the criteria for low risk of persistent/recurrent disease (thyroid carcinoma confined to the thyroid gland) despite the size of the tumour [4]. For patients at risk of recurrence, ablative therapy with radioiodine I-131 may be necessary [2].

Radio-iodine is administered after thyroidectomy in patients with differentiated thyroid cancer to remove residual normal thyroid tissue providing adjuvant therapy of subclinical micrometastatic disease and/ or to provide treatment of clinically apparent residual or metastatic thyroid cancer [6].

In patients who develop distant metastases, radioiodine therapy is sometimes less effective and other treatments such as systemic chemotherapy (kinase inhibitors), external radiotherapy, percutaneous ethanol injection of cervical lymph node metastases, radiofrequency ablation of cervical, bone and lung metastases as well as palliative embolisation of bone metastases are preferred [4].

Given the tumour recurrence in our patient, treatment with radioactive iodine was decided on a palliative basis given the systemic extension found, and he was not considered a candidate for more aggressive treatment.

With this clinical case we wish to highlight how difficult and prolonged it can be to diagnose such a frequent and potentially fatal pathology in a haemodiaysis patient, despite assessing these patients at least 2 or 3 times a week and subjecting them to multiple complementary examinations.

Cancer is the second leading cause of death and morbidity in Europe (3.7 million new cases each year) and the median age of patients at diagnosis is 65 years old.

Today more than ever, if we take into account the increase in the average age of our dialysis patients, and the improvement of techniques leading to an increasing survival of these patients, the close cancerkidney connection explains the need to develop onconephrology as a subspecialty with faster and more efficient diagnostic and therapeutic protocols.

\section{Conflict of Interests}

None.

\section{References}

1. Maisonneuve P, Agodoa L, Gellert R, Stewart JH, Buccianti G, et al. (1999) Cancer in patients on dialysis for end-stage renal disease: an international collaborative study. Lancet 354: 93-99.

2. Gallegos-Villalobos A, García-López F, Escalada C, Ortiz JJ, Cardona $\mathrm{J}$, et al. (2014) Uso de yodo radiactivo I-131 y monitorización de radiactividad en pacientes con enfermedad renal crónica en hemodiálisis. Nefrologia 34: 317-322.

3. Myung J, Choi JH, Yi JH, Kim I (2020) Cancer incidence according to the National Health Information Database in Korean patients with end-stage renal disease receiving hemodialysis. Korean J Intern Med 35: 1210-1219.

4. Tuttle RM (2020) Differentiated thyroid cancer: Overview of management. UpToDate.

5. Mazzaferri EL, Jhiang SM (1994) Long-term impact of initial surgical and medical therapy on papillary and follicular thyroid cancer. Am J Med 97: 418-428.

6. Tuttle RM (2020) Papillary thyroid cancer: Clinical features and prognosis. UpToDate. 